\title{
Uptake of Bile Acid into Calcium-Induced Alginate Gel Beads Containing $\beta$-Chitosan Weak Acid Salt
}

\author{
Yoshifumi Murata ${ }^{*}$, Kyoko Kofuji ${ }^{1}$, Norihisa Nishida², Ryosei Kamaguchi ${ }^{2}$ \\ ${ }^{1}$ Faculty of Pharmaceutical Science, Hokuriku University, Kanazawa, Japan \\ ${ }^{2}$ Morishita Jintan Co. Osaka Technocenter, Hirakata, Japan \\ Email: ${ }^{*}$-murata@hokuriku-u.ac.jp
}

Received 5 February 2014; revised 15 March 2014; accepted 26 March 2014

Copyright (C) 2014 by authors and Scientific Research Publishing Inc.

This work is licensed under the Creative Commons Attribution International License (CC BY). http://creativecommons.org/licenses/by/4.0/

(c) (i) Open Access

\begin{abstract}
Recently, potential applications for $\beta$-chitosan $(\beta$-CS) have been examined. In the present study, calcium-induced alginate gel beads (Alg-Ca) containing weak acid salts of $\beta$-CS were prepared and examined with regard to their ability to adsorb bile acids in vitro. More than $70 \%$ of taurocholate dissolved in solution was taken up by Alg-Ca containing $100 \mathrm{mg} \beta$-CS, sim. ilar to the degree of uptake observed with Alg-Ca containing $\alpha$-CS salt. The adsorption of bile acid was affected by the absolute amount of $\beta$-CS and/or the acid concentration of the preparation. A secondary bile acid, taurodeoxycholate, was also adsorbed by Alg-Ca containing weak acid salts of $\beta$-CS. Therefore, $\beta$-CS might be used to adsorb bile acids within the gastrointestinal tract in the same manner as an anion-exchange resin, and thus serve as a complementary means by which to prevent hyperlipidemia.
\end{abstract}

\section{Keywords}

\section{$\beta$-Chitosan; Bile Acid Adsorption; Alginate Gel Bead; Hyperlipidemia}

\section{Introduction}

Chitin and chitosan (CS) have been utilized in the food and/or medicinal industry because of their biocompatibility and/or biodegradability. Two crystal forms of chitin, $\alpha$-chitin and $\beta$-chitin, are best understood and structural differences between these natural polysaccharides result in different chemical reactivities [1]. Generally, $\alpha$-chitin is extracted from crab or shrimp shell, and $\beta$-chitin from squid pen. CS is a cationic polymer prepared by N-deacetylation of chitin, thus $\alpha$-CS and $\beta$-CS are obtained from $\alpha$-chitin and $\beta$-chitin, respectively. The properties of $\alpha$-CS and $\beta$-CS,

\footnotetext{
${ }^{*}$ Corresponding author.
} 
such as water-solubility and/or anti-microbial activity, also differ [2] [3]. Various chitosan-based drug delivery systems have been investigated [4]-[6]. Most studies to date have examined $\alpha$-CS since a number of commercial preparations of $\alpha$-CS are available.

Though CS is not hydrolyzed by human digestive enzymes, it is an attractive material for drug development due to its biological effects or adsorptive capacity in the gastrointestinal tract when taken orally [7]. For example, it is widely recognized that oral administration of $\alpha$-CS reduces serum cholesterol levels [8] [9]. One mechanism by which this occurs is via adsorption of bile acids within the intestinal tract. We have previously reported that a weak acid salt of $\alpha$-CS adsorbs bile acids through electrostatic interactions and that oral administration of the salt reduces serum cholesterol levels in rats [10] [11]. We have also shown that a polymer matrix containing $\alpha$-CS salt adsorbs bile acids by the same mechanism [12].

Allergic reactions, including anaphylaxis, following ingestion of $\alpha$-chitin or $\alpha$-CS can be problematic. The antigenicity of $\alpha$-chitin or $\alpha$-CS is primarily attributable to proteins such as tropomyosin or arginine kinase that contaminate the $\alpha$-chitin extract during processing [13] [14]. For this reason, $\beta$-chitin and $\beta$-CS are gaining more interest due to their low antigenicity. A potential role for $\beta$-CS in applications related to food hygiene is being examined [15] [16]. In the present study, we tried to devise a dosage form of $\beta$-CS salt for incorporation into calcium-induced alginate gel beads (Alg-Ca). The ability to take up bile acids secreted into the human intestine was examined in vitro.

\section{Experimental}

\subsection{Materials}

Two $\beta$-CSs, $\beta$-CS1 (deacetylation (DA): 96\%, Yaegaki Bio-industry Inc., Himeji, Japan) and $\beta$-CS2 (DA: above 90\%, FAP Japan, Tokyo, Japan), as well as $\alpha$-CS (DA: 94\%, Koyo Chemical Co., Tokyo, Japan), were used. Sodium alginate (300 cps) and four bile acids, sodium taurocholate (TCA), glycocholate (GCA), taurodeoxycholate (TDCA), and taurochenodeoxycholate (TCDA), were purchased from Nacalai Tesque (Kyoto, Japan). Six reagent grade weak acids were also used to prepare the CS salt: lactic, malic, citric, sorbic, nicotinic, and ascorbic acid.

\subsection{Preparation of the CS Lactic Acid Salt}

One gram of CS powder and $1 \mathrm{~g}$ of lactic acid were added to $18 \mathrm{~g}$ of demineralized water and the mixture stirred until the CS powder dissolved. Ethanol $(180 \mathrm{~mL})$ was added to the viscous CS solution, followed by vigorous stirring. The suspension was then centrifuged at $3500 \mathrm{rpm}(1370 \times \mathrm{g})$ for $10 \mathrm{~min}$. The precipitate was collected and washed five times with ethanol, then dried on a dish. It was desiccated under a vacuum in the presence of $\mathrm{P}_{2} \mathrm{O}_{5}$.

\subsection{Preparation of Alg-Ca Containing $\beta$-CS Salt}

Alg-Ca containing $\beta$-CS salt were prepared as follows: $0.5 \mathrm{~g}$ of $\beta$-CS was dispersed in $9.5 \mathrm{~g}$ of $1.5(\mathrm{w} / \mathrm{w}) \%$ sodium alginate solution with agitation. This solution $(2 \mathrm{~g})$ was dropped into $10 \mathrm{ml}$ of $0.1 \mathrm{M} \mathrm{CaCl}_{2}$ containing weak acid and left to stand at $37^{\circ} \mathrm{C}$ for $1 \mathrm{~h}$. Next, the spherical hydrogel beads were transferred to $20 \mathrm{ml}$ of demineralized water, which was incubated in a shaker incubator (SI-300; As One Co., Osaka, Japan) at $37^{\circ} \mathrm{C}$ for 1 $\mathrm{d}$. The Alg-Ca (hydrogel beads) were then taken out and dried at $37^{\circ} \mathrm{C}$ for $1 \mathrm{~d}$ on a dish, followed by vacuum treatment in a desiccator in the presence of $\mathrm{P}_{2} \mathrm{O}_{5}$. In this experiment, uptake of $\beta$-CS salt into Alg-Ca was observed by stereoscopic microscopy (Carton Co., Tokyo, Japan), for which a PC was directly connected to a USB camera (MeCan Imaging Inc., Fujimino, Japan).

\subsection{Test of Bile Acid Adsorption}

Fifteen milliliters of a $2 \mathrm{mM}$ bile acid solution were placed into $50 \mathrm{ml}$ vials and maintained at $37^{\circ} \mathrm{C}$. Dried Alg-Ca corresponding to $2 \mathrm{~g}$ of hydrogel were added to the tubes and shaken at $300 \mathrm{rpm}$ in a shaker incubator at $37^{\circ} \mathrm{C}$. A $180 \mu \mathrm{l}$ aliquot of each solution was removed periodically to determine the amount of bile acid by HPLC analysis.

\subsection{Determination of Bile Acids}

The HPLC system had a pump (LC-10AS; Shimadzu, Kyoto), a packed column (Mightysil RP-18 GP, 150 mm 
× 4.6 mm, Kanto Chem. Co., Tokyo), a detector (SCL-10A; Shimadzu), and an auto-injector (SIL-10A; Shimadzu). HPLC was conducted at ambient temperature using an eluent containing methanol, $30 \mathrm{mM}$ phosphate buffer (pH 3.4), and acetonitrile (6:3:1), at a flow rate of $0.8 \mathrm{ml} / \mathrm{min}$. The detector wavelength was set at $220 \mathrm{~nm}$. Typical chromatograms of bile acids were shown in Figure 1. The amount of bile acid taken up into the Alg-Ca was calculated based on the amount of bile acid added and that remaining at sampling time. All tests were performed in triplicate. Where necessary, data were compared using Student's $t$-test and their difference was inferred as significance when $p<0.01$.

\section{Results and Discussion}

The lactic acid salt of $\beta$-CS1 obtained in this study was a yellow powder which dissolved in water. Though the CS salt swelled in $2 \mathrm{mM}$ of TCA, it did not dissolve. This property was also observed with the $\alpha$-CS lactic acid salt. A precipitate gradually appeared after $5 \mathrm{ml}$ of $1 \% \beta$-CS1 salt solution was added to an equal volume of 2 $\mathrm{mM}$ TCA at $37^{\circ} \mathrm{C}$. This illustrates that an interaction may occur between $\beta$-CS1 and bile acid sodium salts within solution.

Alg-Ca incorporating $\beta$-CS formed as soon as the sodium alginate was dropped into the $\mathrm{CaCl}_{2}$ solution containing weak acid such as lactic acid. Following this, formation of the $\beta$-CS lactic acid salt was progressed by penetration of lactic acid into the alginate gel matrix, as shown in Figure 2. When the hydrogel beads containing $100 \mathrm{mg} \beta$-CS1 were transferred to a solution containing $30 \mu \mathrm{mol} \mathrm{TCA}$, they adsorbed bile acid $(18.8 \pm 0.1 \mu \mathrm{mol}$ was taken up into Alg-Ca after $120 \mathrm{~min}$ ). Uptake was not observed, however, when Alg-Ca containing $\beta$-CS1 was prepared with acid-free $\mathrm{CaCl}_{2}$ solution. In this circumstance, the dried gel beads were observed to swell in solution taking up $21.3 \pm 0.2 \mu \mathrm{mol}$ of TCA at $60 \mathrm{~min}$, although the initial rate of uptake was less than that of hydrogel. The uptake behavior of bile acid was similar for Alg-Ca containing $\alpha$-CS lactic acid salt. A similar phenomenon was also observed for Alg-Ca containing the lactic acid salt of $\beta$-CS2, for which uptake of TCA was $17.8 \pm 0.4 \mu \mathrm{mol}$ at $60 \mathrm{~min}$.

The uptake of bile acid into Alg-Ca varied with lactic acid concentration within the $\mathrm{CaCl}_{2}$ solution, as shown in Figure 3. A dose-dependent relationship between the rate of uptake of bile acid and the acid concentration was observed. The absolute amount of TCA uptake depended on the amount of available $\beta$-CS. More than $70 \%$ of the bile acid initially dissolved in solution was incorporated into Alg-Ca containing $100 \mathrm{mg} \beta$-CS1, as shown in Figure 4. These results illustrate that uptake is primarily attributable to an ion-exchange reaction between the

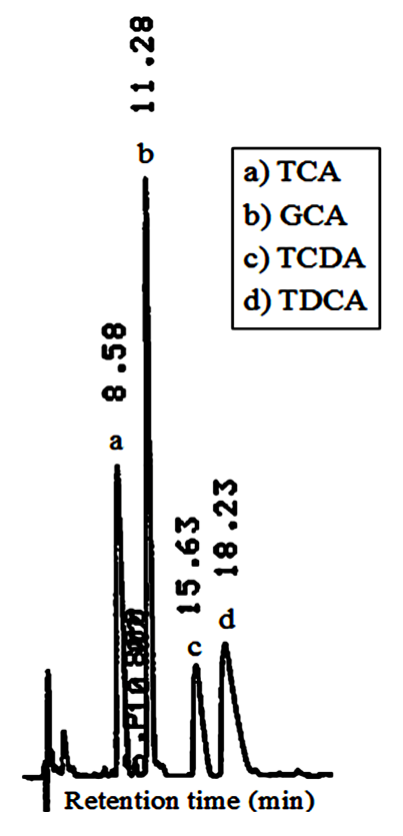

Figure 1. Chromatograms of bile acids (20 nmol) obtained from HPLC determination. 


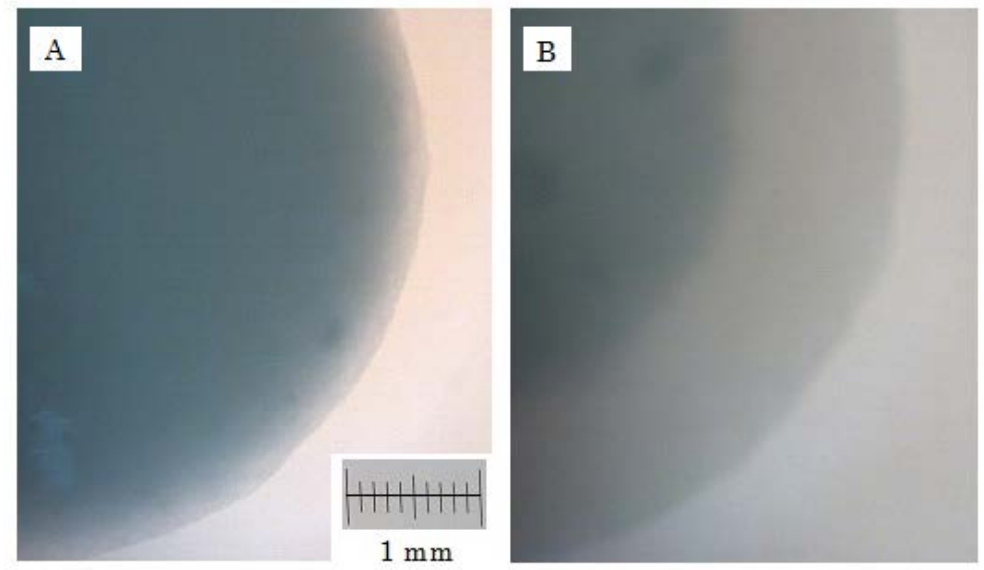

Figure 2. Stereoscopic photomicrograph of Alg-Ca containing $100 \mathrm{mg} \beta$-CS1 in $0.1 \mathrm{M} \mathrm{CaCl}_{2}$ solution containing $1 \%$ lactic acid at $37^{\circ} \mathrm{C}$. (A) $0 \mathrm{~min}$; (B) 60 $\min$.

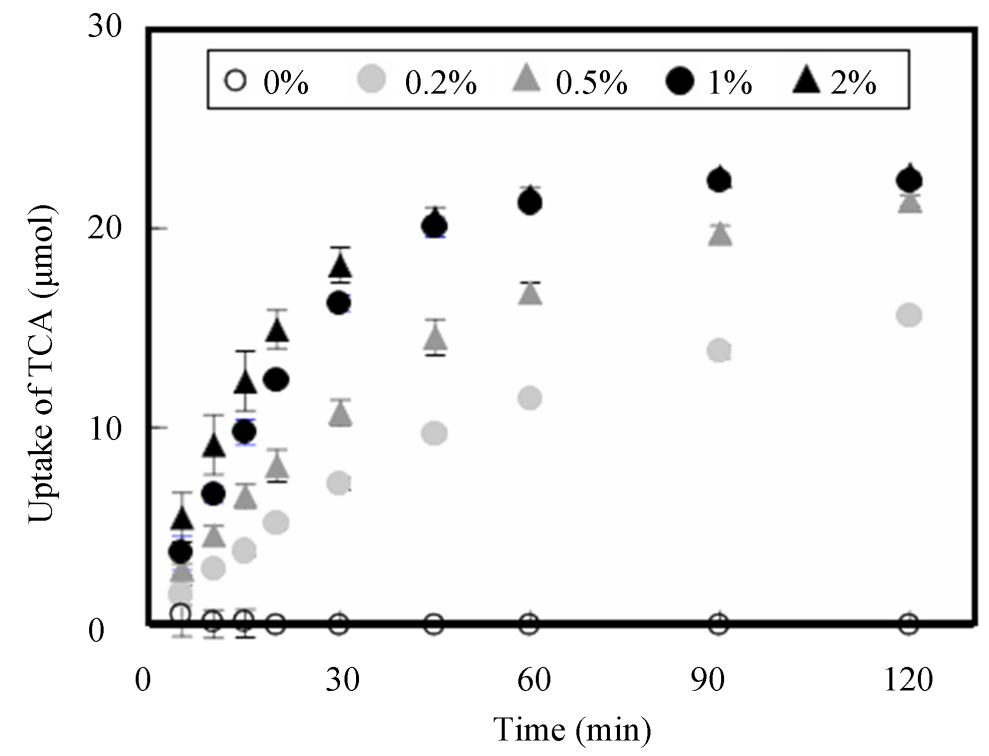

Figure 3. Effect of lactic acid concentration on uptake of TCA into Alg-Ca containing $100 \mathrm{mg} \beta$-CS1.

bile acid and lactic acid.

Figure 5 shows the various profiles of TCA uptake by Alg-Ca containing $\beta$-CS treated with various weak acids. CS salt prepared with not only lactic acid but also malic acid or citric acid was able to adsorb bile acid. Similar uptake profiles of bile acid were observed for Alg-Ca containing $\beta$-CS treated with sorbic acid, nicotinic acid and ascorbic acid, which are utilized as food additives. In any case, similar amounts of TCA (19 - 22 $\mu \mathrm{mol} / \mathrm{sample}$ ) were taken into Alg-Ca at $60 \mathrm{~min}$. Primary bile acids, including TCA, GCA and TCDA, are secreted into the human gastrointestinal tract, while secondary bile acids, for example TCDA, are produced by enteric bacteria. All of these bile acids can be adsorbed by Alg-Ca containing $\beta$-CS1, and more than 90\% of TDCA, in particular, becomes adsorbed by 30 min, as shown in Table 1 .

Bile acids are formed in the liver from cholesterol and 12 - $36 \mathrm{mg}$ /day are secreted into the intestinal tract [17]. Adsorption of bile acids due to the presence of an anion-exchange resin in the intestinal tract inhibits their enterohepatic circulation and reduces plasma cholesterol levels. It is presumed that molecular weight and degree of deacetylation are important factors affecting bile acid adsorption by $\beta$-CS. In the present study, a commercial preparation of $\beta$-CS prepared from a weak acid salt was used and Alg-Ca was selected as the vehicle in order to 


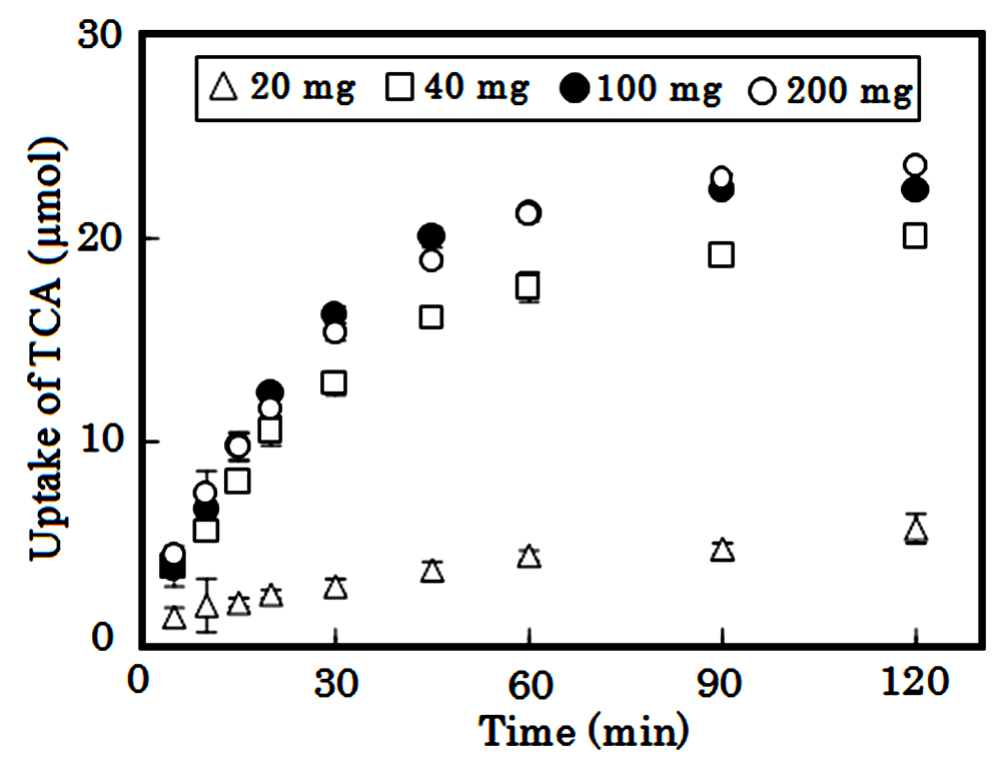

Figure 4. Effect of $\beta$-CS1 amount on uptake of TCA into Alg-Ca treated with $1 \%$ lactic acid.

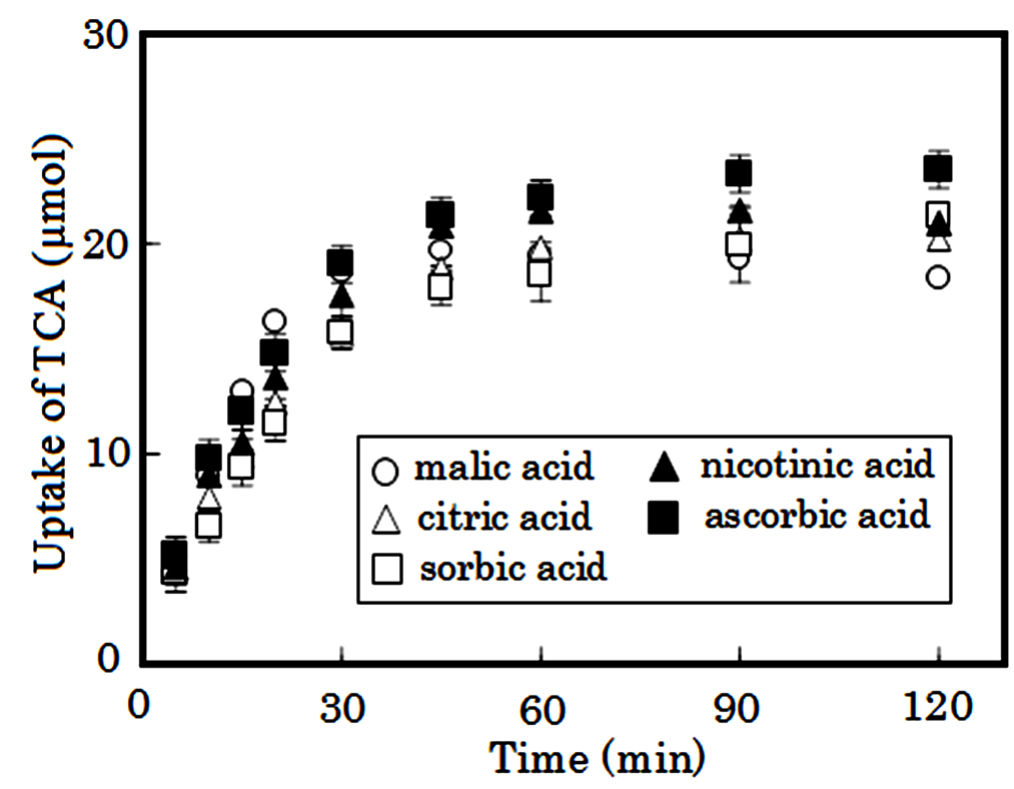

Figure 5. Uptake of TCA into Alg-Ca containing $100 \mathrm{mg} \beta$-CS1 treated with $1 \%$ weak acid.

Table 1. Amount of bile acid taken up into Alg-Ca containing $100 \mathrm{mg} \beta$-CS1 treated with $1 \%$ lactic acid (n = 3, mean \pm S.D.).

\begin{tabular}{ccccc}
\hline Time $(\min )$ & TCA $(\mu \mathrm{mol})$ & GCA $(\mu \mathrm{mol})$ & TCDC $(\mu \mathrm{mol})$ & TDCA $(\mu \mathrm{mol})$ \\
\hline 30 & $16.2 \pm 0.4$ & $15.2 \pm 0.5$ & $26.7 \pm 0.2$ & $27.9 \pm 0.1$ \\
60 & $21.3 \pm 0.2$ & $20.4 \pm 0.4$ & $27.4 \pm 0.1$ & $29.0 \pm 0.3$ \\
\hline
\end{tabular}

provide an oral form of $\beta$-CS powder that could be swallowed. Alg-Ca containing $\beta$-CS salt adsorbed bile acid to a similar extent as Alg-Ca containing $\alpha$-CS salt. The significant difference was not seen statistically $(p<0.01)$ on the uptake amounts of TCA at $60 \mathrm{~min}$ or $120 \mathrm{~min}$ between these vehicles. In light of the fact that all of the 
materials here examined are safe for oral consumption, this vehicle may serve as a complementary agent targeted at preventing hyperlipidemia.

\section{Acknowledgements}

This work was supported in part by a grant from The OTC Self-Medication Promotion Foundation (2013).

\section{References}

[1] Fan, Y., Saito, T. and Isogai, A. (2008) Preparation of Chitin Nanofibers from Squid Pen $\beta$-Chitin by Simple Mechanical Treatment under Acid Conditions. Biomacromolecules, 9, 1919-1923. http://dx.doi.org/10.1021/bm800178b

[2] Jung, J. and Zhao, Y. (2013) Impact of the Structural Differences between $\alpha$ - and $\beta$-Chitosan on Their Depolymerizing Reaction and Antibacterial Activity. Journal of Agricultural Food Chemistry, 61, 8783-8789. http://dx.doi.org/10.1021/jf4018965

[3] Subhapradha, N., Ramasamy, P., Shanmugam, V., Madeswaran, P., Srinivasan, A. and Shanmugam, A. (2013) Physicochemical Characterization of $\beta$-Chitosan from Sepioteuthis Lessoniana Gladius. Food Chemistry, 141, 907-913. http://dx.doi.org/10.1016/j.foodchem.2013.03.098

[4] Hu, L., Sun, Y. and Wu, Y. (2013) Advances in Chitosan-Based Drug Delivery Vehicles. Nanoscale, 5, 3103-3111. http://dx.doi.org/10.1039/c3nr00338h

[5] Shukla, S.K., Mishra, A.K., Arotiba, O.A. and Mamba, B.B. (2013) Chitosan-Based Nanomaterials: A State-of-the-Art Review. International Journal of Biological Macromolecules, 59, 46-58. http://dx.doi.org/10.1016/j.ijbiomac.2013.04.043

[6] Ragelle, H., Vandermeulen, G. and Preat, V. (2013) Chitosan-Based siRNA Delivery Systems. Journal of Controlled Release, 172, 207-218. http://dx.doi.org/10.1016/j.jconrel.2013.08.005

[7] Pahwa, R., Saini, N., Kumar, V. and Kohli, K. (2012) Chitosan-Based Gastroretentive Floating Drug Delivery Technology: An Update Review. Expert Opinion on Drug Delivery, 9, 525-539. http://dx.doi.org/10.1517/17425247.2012.673581

[8] Zhang, J., Liu, J., Li, L. and Xia, W. (2008) Dietary Chitosan Improves Hypercholesterolemia in Rats Fed High-Fat Diets. Nutrition Research, 28, 383-390. http://dx.doi.org/10.1016/j.nutres.2007.12.013

[9] Liu, S.H., He, S.P. and Chiang, M.T. (2012) Effect of Long-Term Feeding of Chitosan on Postprandial Lipid Responses and Lipid Metabolism in a High-Sucrose-Diet-Impaired Glucose-Tolerant Rat Model. Journal of Agricultural Food Chemistry, 60, 4306-4313. http://dx.doi.org/10.1021/jf300792b

[10] Murata, Y., Kojima, N. and Kawashima, S. (2003) Function of a Chitosan-Orotic Acid Salt in the Gastrointestinal Tract. Biological \& Pharmaceutical Bulletin, 26, 687-690. http://dx.doi.org/10.1248/bpb.26.687

[11] Murata, Y., Nagaki, K., Kofuji, K., Sanae, F., Kontani, H. and Kawashima, S. (2006) Adsorption of Bile Acid by Chitosan Salts Prepared with Cinnamic Acid and Analogue Compounds. Journal of Biomaterials Science, Polymer Edition, 17, 781-789. http://dx.doi.org/10.1163/156856206777656517

[12] Murata, Y., Nagaki, K., Kofuji, K. and Kishi, T. (2010) Functions of Chitosan-Ferulic Acid Salt for Prevention of Hypertension. Food Science and Technology Research, 16, 437-442. http://dx.doi.org/10.3136/fstr.16.437

[13] Rosmilah, M., Shahnaz, M., Zailatul, H.M., Noormalin, A. and Normilah, I. (2012) Identification of Tropomyosin and Arginine Kinase as Major Allergens of Portunus pelagicus (Blue Swimming Crab). Tropical Biomedicine, 29, 467-478.

[14] Misnan, R., Murad, S., Yadzir, Z.H. and Abdullah, N. (2012) Identification of the Major Allergens of Charybdis feriatus (Red Crab) and Its Cross-Reactivity with Portunus pelagicus (Blue Crab). Asian Pacific Journal of Allergy and Immunology, 30, 285-293.

[15] Bae, M.-J., Shin, H.S., Kim, E.-K., Kim, J. and Shon, D.-H. (2013) Oral Administration of Chitin and Chitosan Prevents Peanut-Induced Anaphylaxis in a Murine Food Allergy Model. International Journal of Biological Macromolecules, 61, 164-168. http://dx.doi.org/10.1016/j.ijbiomac.2013.06.017

[16] Jung, J., Cavender, G. and Zhao, Y. (2014) The Contribution of Acidulant to the Antibacterial Activity of Acid Soluble $\alpha$ - and $\beta$-Chitosan Solutions and Their Films. Applied Microbiology and Biotechnology, 98, 425-435. http://dx.doi.org/10.1007/s00253-013-5334-7

[17] Martin, C.C. (1982) The Liver, Biology and Pathobiology. Arias, A., Ed., Raven Press, New York. 


\section{Abbreviations}

Alg-Ca; Calcium-Induced Alginate Gel Beads

CS; Chitosan

DA; Deacetylation

GCA; Glycocholate

TCA; Taurocholate

TCDA; Taurochenodeoxycholate

TDCA; Taurodeoxycholate 